\title{
VERIFICAÇÃO DA OCORRÊNCIA DE PLANTAS COM POTENCIAL DE TOXICIDADE NOS JARDINS DO CAMPUS MOOCA DA UNIVERSIDADE SÃO JUDAS TADEU (SÃO PAULO/SP)
}

\author{
Fabiana Pereira Santos ${ }^{1}$ \\ André Rinaldi Fukushima ${ }^{2}$ \\ Oriana Aparecida Fávero ${ }^{3}$
}

\begin{abstract}
RESUMO
Considerando que cerca de $60 \%$ dos casos de intoxicação no Brasil são por plantas, por vezes ornamentais, este trabalho teve como objetivo verificar se as plantas que compõe os jardins do Campus Mooca da Universidade São Judas Tadeu (USJT) apresentam algum potencial de toxicidade com base em estudos científicos. Para isso foi realizado um levantamento das espécies vegetais, que ocorrem nos canteiros ornamentais do Campus, bem como, sua identificação botânica, para verificação, em obras científicas, do potencial risco que as espécies mais frequentes podem oferecer às pessoas. Verificou-se a ocorrência de 19 canteiros vegetados no Campus Mooca da USJT, para os quais foram elaborados croquis com a plotagem de ocorrência e distribuição dos vegetais identificados. Nestes canteiros ocorrem 209 populações vegetais, as quais pertencem a cerca de 100 espécies diferentes das quais 26 espécies compõem mais da metade $(55,50 \%)$ das populações nos canteiros. Dentre estas 26 espécies, 14 (30,59\% das populações) apresentam, de acordo com literatura científica, potencial de toxicidade, especialmente se ingeridas diretamente ou se exsudatos que as constituem (como o látex, por exemplo) entrarem em contato com mucosas. Considerando que o local de estudo apresenta enorme fluxo de pessoas entre funcionários, alunos e visitantes (em torno de 25.000 especialmente no período noturno) estes resultados podem contribuir para escolha de medidas de planejamento paisagístico que ofereçam maior segurança às pessoas.
\end{abstract}

PALAVRAS-CHAVE: áreas verdes, plantas ornamentais, toxicidade.

\section{PLANS OCCURRENCE OF CHECKING WITH POTENTIAL TOXICITY IN CAMPUS UNIVERSITY MOOCA GARDENS ST. JUDE ( SÃO PAULO / SP )}

\begin{abstract}
Whereas about $60 \%$ of cases of intoxication by plants in Brazil are by ornamental plants, this study aimed to verify if the plants that make up the gardens of the Campus Mooca at the Universidade São Judas Tadeu (USJT) have some potential for toxicity based on others scientific studies. For this it was performed a survey of plant species in flower beds at the Campus, as well as, its botanical identification, with base in scientific literature to verify the potential risk that the most frequent species can offer to the health of the people. It has been found the occurrence of 19 flower beds vegetated in Campus Mooca at the USJT, and then sketches were drawn up with the occurrence of plotting and

\footnotetext{
${ }^{1}$ Cursando Farmácia, Universidade São Judas Tadeu. fabyanasaantos@hotmail.com

${ }^{2}$ Farmacêutico, Mestre em toxicologia e análises toxicológicas (FCF/USP), Doutorado em andamento pela Faculdade de Medicina Veterinária e Zootecnia (USP); fukushima@usp.br.

${ }^{3}$ Bióloga, Mestre e Doutora em Geografia Humana (FFLCH/USP), professora titular da FCBS/USJT; lulubae@gmail.com
} 
distribution of identified plants. These beds occur 209 plant populations, which belong to about 100 different species of which 26 species make up more than half ( $55.50 \%$ ) of the people in beds. Among the 26 species, 14 (30,59\% of the population) have, according to literature, potential toxicity, especially when directly ingested or if exudates (such as latex, for example) come into contact with mucous membranes. Considering that the place of study presents huge flux of people between staff, students and visitors (around 25,000 especially at night) these results can contribute to choice of landscape planning measures to provide greater security to the health and security of the people.

KEY WORDS: green areas, ornamental plants, toxicity.

\title{
PLANES DE OCURRENCIA DE COMPROBAR CON POTENCIAL TOXICIDAD EN CAMPUS UNIVERSITARIO MOOCA JARDINES SAN JUDAS ( SÃO PAULO / SP )
}

\begin{abstract}
RESUMEN
Considerando que cerca de $60 \%$ de los casos de intoxicación en el Brasil, son por plantas, por veces ornamentales, este trabajo túvo como objetivo verificar si las plantas que componén los jardines de Campus Mooca de la Universidade São Judas Tadeu (USJT), representán algún potencial de tóxicidad com base em lós estudios cientificos. Para esto fué realizado un levantamiento de lãs especies vegetales, que encurrén en lós cánteros ornamentales de Campus, bien como, sú indentificación bótanica, para lãs personas, verificación, en obrás cientificas, del potencial de riésgo que las espécies más frecuentes pueden oferecer a las personas. Se aberigúo que La occorrencia de 19 cánteros vegetados en el Campus Mooca de USJT, para los cuales, fuerón elaborados croquis com la plotagem de lá ocorrência y distribuición de vegetales identificados. En estes cánteros ocurrén 209 populaciones vegetales, en las cúales pertencen a cerca de 100 especies diferentes de las cuáles 26 especies componén más de la mitad (55.50\%) de las poblaciones en los canteros. Entre estas 26, especies, 14 (30,59\% de las poblaciones) representán de acuerdo con la literatura cientifica, potenciál de toxicidad, especialmente si ingeridos directamente o cuando ocorre directamente ingestión de los exudados que son (como el látex por ejemplo) entrarán en contacto con la mucosas. Considerando que el local de estudio representa enorme fluxo de personas entre funcionarios, alumnos y visitantes (en torno de 25.000, especialmente en el período noturno. Estos resultados pueden contribuir para elegir de medidas de planeamiento paisajistico que ofrecén mayor seguridad a las personas.
\end{abstract}

PALABRAS CLAVE: areas verdes, plantas ornamentales, toxicidad.

\section{INTRODUÇÃO}

Nas áreas urbanizadas a presença de vegetação oferece inúmeros benefícios à saúde física e mental da população tais como: melhoria da qualidade do ar, oferta de recursos alimentícios, bem-estar psicológico; todos incrementando significativamente a qualidade ambiental e de vida da população nestas áreas (SALATINO, 2001; NUCCI, 2008).

O paisagismo tem como objetivo "melhorar a qualidade visual e ambiental de áreas externas de edificações diversas como as de instituições de ensino" (BIONDI 


\section{Periódica Eletranica \\ Fórum Ambiental}

da Alta Paulista
Volume 11, Número 08, 2015

Saúde e Saneamento Ambiental

et al., 2008). Compreende a elaboração de projetos que melhorem o conforto ambiental, protegendo o solo contra erosão, a recuperação da vegetação e sua manutenção, com a finalidade de criar melhores condições de lazer (ORTEGA et al., 2008).

Muitas das plantas cultivadas como ornamentais em jardins apresentam pouco conhecimento cientifico sobre seu potencial de uso e toxicidade a população e frequentemente podem apresentar riscos à saúde, como algum principio tóxico, levando a alergias, salivação, diarreia entre outros, assim dificultando o diagnostico e um possível tratamento (FABIANO e FRANCISCO, 2009).

A intoxicação provocada por plantas ornamentais (todas aquelas que são cultivadas pela sua beleza) e medicinais (que podem contribuir com o tratamento e cura de doenças), tem causado graves problemas para a saúde da população e a economia do país, por levar a doenças e acarretar a tratamentos. É a quarta causa de intoxicação no Brasil (MATOS et al., 2011).

Conforme as diretrizes desenvolvidas pela fundação para o desenvolvimento da Educação - EDF, e a Companhia de Construções Escolares do Estado de São Paulo - CONESP, devem-se seguir alguns critérios em relação ao paisagismo nas escolas, dentre eles as plantas devem: apresentar características botânicas apropriadas as exigência da escola; estar adequadas às condições climáticas e geológicas da região; ser de fácil reprodução e manutenção; ser nativa com caráter simbólico; e não devem apresentar espinhos e nem princípios tóxicos ou alergênicos (BIONDI et al., 2008).

No Brasil, o ministério da saúde relata que há cerca de 2.000 casos de intoxicação por plantas, dos quais 5\% ocorrem em animais e 95\% ocorrem com humanos, sendo destes $20 \%$ de intoxicação em adultos e $70 \%$ em crianças e o restante dos casos em idosos e adolescentes (FABIANO e FRANCISCO, 2009).

Como exemplos de plantas, muito utilizadas em ornamentação urbana, que oferecem riscos à população, pode-se destacar: a azaleia (Rhododendron spp, Ericaceae) que apresenta, em toda a planta, andromedotoxina, que se ingerida causa salivação, cólicas e paralisia respiratória (BARCELLOS, 2012); e a comigoninguém-pode (Diffenbachia spp), planta utilizada como ornamental no Brasil, porém originária da Índia, que por possuir uma seiva leitosa com grandes quantidades de cristais de oxalato de cálcio e glicosídeos, se ingerida, pode causar edema da 
língua, salivação, úlcera, vômitos entre outros sintomas de intoxicação (SILVA e TAKEMURA, 2006).

O Sistema Nacional de Informações Toxico-Farmacológicas em parceria com a fundação Oswaldo Cruz relatou que cerca de $60 \%$ dos casos de intoxicação no Brasil, ocorrem por plantas devido o desconhecimento do potencial toxico (FABIANO e FRANCISCO, 2009).

Segundo Silva (2009) ambientes institucionais com grande fluxo de pessoas (como o Campus Mooca da Universidade São Judas Tadeu) são locais onde tornase extremamente necessário estudo específico sobre plantas tóxicas nas áreas verdes tanto para melhor investigação das espécies vegetais já implantadas nestes ambientes quanto para diagnosticar com mais eficiência o potencial de incidentes com plantas e auxiliar em melhores planejamentos paisagísticos destes espaços, bem como na prevenção de intoxicações.

\section{OBJETIVOS}

\subsection{OBJETIVO GERAL}

Considerando que, muitas vezes, plantas ornamentais podem ter toxicidade e oferecer riscos à saúde humana o presente trabalho objetivou verificar dentre as plantas mais frequentes, nos jardins e canteiros com plantas ornamentais da Universidade São Judas Tadeu - Campus Mooca, quais podem oferecer riscos à saúde humana e de animais por apresentar algum tipo de toxicidade, bem como qual é, segundo estudos científicos, seu potencial de toxicidade.

\subsection{OBJETIVOS ESPECÍFICOS}

Levantamento das espécies que ocorrem nos canteiros de plantas ornamentais da USJT e verificação de quais são as predominantes.

Verificação, por meio de revisão bibliográfica, se as espécies, mais frequentes na área de estudo, apresentam: algum estudo científico que indique qualquer potencial de toxicidade e, se sim, qual é o agente (químico ou estrutural), que pode causar a intoxicação ou dano, bem como quais sintomas são desencadeados do processo de intoxicação. 


\section{METODOLOGIA}

\subsection{LOCALIZAÇÃO DA ÁREA DE ESTUDO}

A cidade de São Paulo apresenta cerca de 11 milhões de habitantes segundo o Censo de 2010, com município de área $1.530 \mathrm{Km}^{2}$. A capital paulista é considerada o centro financeiro da América latina (SAMPAIO, 2014)

O Campus Mooca da USJT localiza-se próximo à região central de São Paulo/ SP, no Distrito da Mooca da Subprefeitura da Mooca como mostra a Figura 1. Figura 1 : Localização do Campus Mooca da USJT na Cidade de São Paulo.
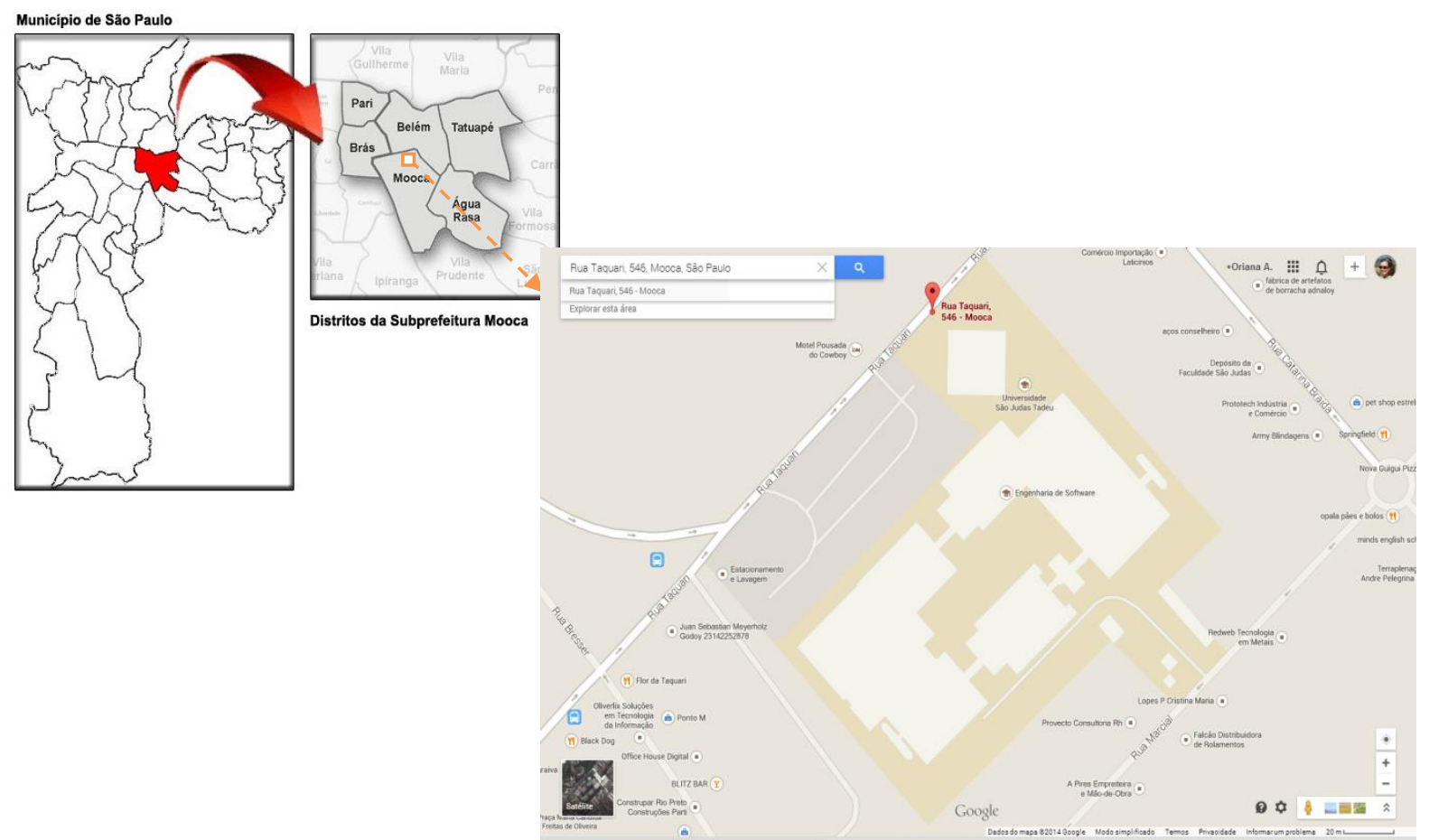

(Org.: FÁVERO, 2014; Fontes: http://www.prefeitura.sp.gov.br/cidade/secretarias/subprefeituras /mooca/mapas/ e https://www.google.com.br/maps/).

A USJT oferece dezenas de cursos no Campus Mooca o que movimenta mais de 25.000 pessoas, entre alunos, funcionários e visitantes, neste campus todos os dias.

\subsection{PROCEDIMENTOS}

A pesquisa contou com duas etapas principais: primeiramente pesquisa exploratória para verificação dos canteiros e plantas ornamental neles contidos seguida da identificação das plantas; e finalmente, pesquisa dedutiva indireta para verificação, em obras científicas, do potencial de toxicidade das espécies mais frequentes nos canteiros.

Para o levantamento florístico foram elaborados croquis, com a plotagem dos canteiros ornamentais, do Campus Mooca da USJT, e nestes primeiramente foi 
realizado levantamento das espécies vegetais ornamentais que neles ocorrem (inventário da flora do Campus).

Em seguida foi realizada a identificação botânica dos indivíduos encontrados em cada canteiro e esquematização de sua distribuição nos mesmos.

$\mathrm{Na}$ identificação utilizou-se a comparação de características morfológicas (tipos de folhas, ramificação, casca do tronco, tipos florais e frutíferos) e fenológicas (caducismo, floração e frutificação, etc.), observadas e fotografadas nas plantas, com a descrição detalhada, e em fotografias de boa qualidade gráfica, destas características, em manuais, amplamente utilizados e já consagrados, de identificação de plantas, da autoria de Lorenzi e colaboradores (1999 e 2008).

Para a revisão de toxicidade das espécies utilizou-se como palavras-chave os nomes específicos das plantas e o termo toxicidade e realizou-se busca em indexadores de artigos científicos da internet como o Scielo Brasil e Sciensedirect.

\section{RESULTADOS}

Verificou-se a ocorrência de 19 canteiros vegetados no Campus Mooca da USJT, distribuídos principalmente na entrada principal deste Campus e no vão livre interno, entre os prédios, em seu primeiro andar.

Foram elaborados croquis para os 19 canteiros com a plotagem de ocorrência e distribuição dos vegetais identificados. A Figura 2 é exemplo de um croquis de um canteiro com a ocorrência e distribuição das espécies vegetais ornamentais.

Do levantamento florístico verificou-se a ocorrência de 209 populações vegetais, espalhadas nos 19 canteiros, as quais pertencem a cerca de 100 espécies vegetais diferentes, que foram codificadas com números sequenciais e cujas informações de ocorrência nos canteiros foram organizadas em um quadro geral do qual destacou-se, no Quadro 1, as espécies mais frequentes nos canteiros. 
Figura 2: Croquis do Canteiro C3- Canteiro entre as duas rampas 2, localizado no vão livre interno, entre os prédios, no primeiro andar do Campus Mooca da USJT.

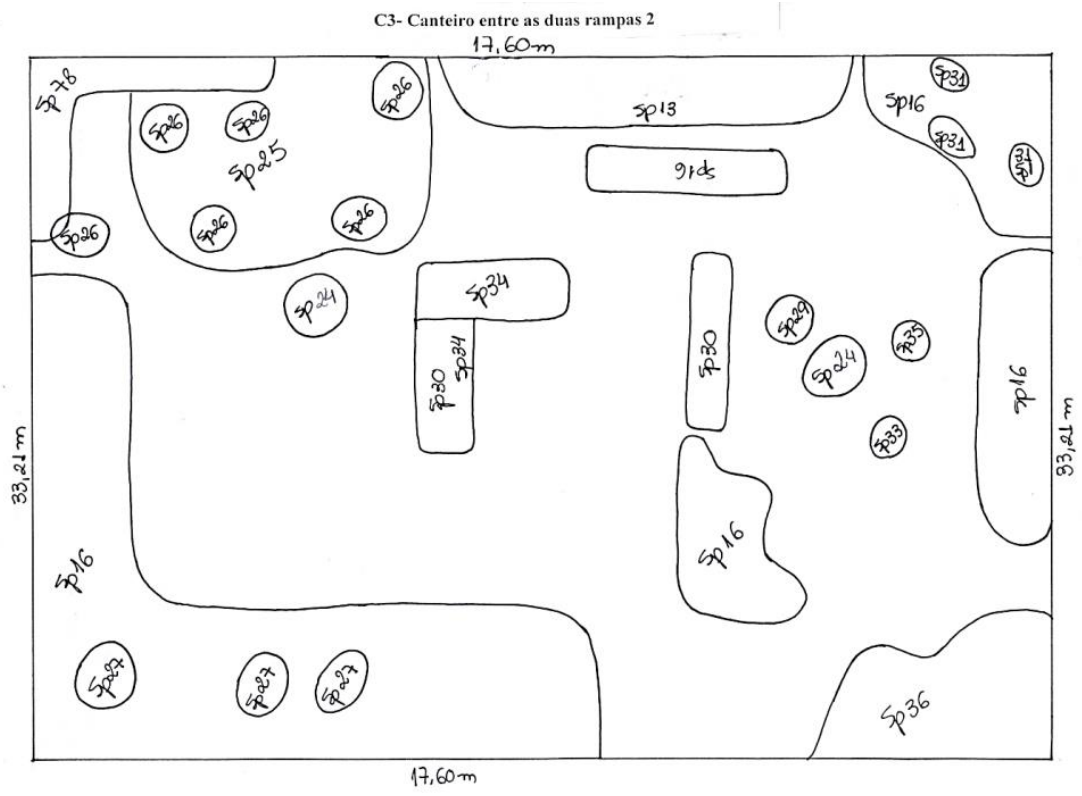

(Org.: SANTOS, 2014)

Nota-se, no Quadro 1, que das cerca de 100 espécies que ocorrem nos canteiros da USJT, 26 compõem mais da metade (55,50\%) das populações nos canteiros, destacando-se entre estas: a palmeira-areca (Dypsis lutescens), espécie mais comum nos canteiros, com 4,78\% de ocorrência; a grama- amendoim (Arachis repens), segunda espécie mais frequente com 4,31\% de ocorrência; as Araceae, orelha-de-elefante (Syngonium spp) e lírio-da-paz (Spathiphyllum wallisii), com $3,35 \%$ de ocorrência cada, e a jibóia (Scindapsus aureus) (2,87\% de ocorrência); e ainda a azaléia (Rhododendon spp) (2,87\% de ocorrência).

Para 12 das 26 espécies mais frequentes nos canteiros, não foram encontrados relatos em obras científicas de presença de toxicidade, as duas mais comuns [Dypsis lutescens (palmeira-areca) e Arachis repens (grama-amendoim)], juntamente com: Sphagneticola trilobata (vedélia), Evolvulus sp (azulzinha), Cyclanthus bipartitus (ciclantus), Dracaena fragrans (dracena), Chlorophytum comosum (gravatinha), Eucharis grandiflora (lírio-do-amazonas), Rhapis excelsa (palmeira-rapis), Pilea cadierei (planta-alumínio), Pleomele reflexa (pleomele) e Lagerstroemia indica (resedá); correspondendo a cerca de $24,91 \%$ das populações vegetais nos canteiros. 
Quadro 1: Lista das espécies mais frequentes nos canteiros da USJT, com suas respectivas famílias botânicas e o percentual de ocorrência (\%).

\begin{tabular}{|c|c|c|c|c|}
\hline COD. & Nome Popular & Espécie Vegetal & Família Botânica & $\%$ \\
\hline $\mathbf{1 3}$ & palmeira-areca & Dypsis lutescens (H. Wendl.) Beentje \& & Arecaceae & 4,78 \\
\hline $\mathbf{1 4}$ & Jrama-amendoim & Arachis repens Handro & Leguminosae & 4,31 \\
\hline $\mathbf{9}$ & lírio-da-paz & Spathiphyllum wallisii Regel & Araceae & 3,35 \\
\hline $\mathbf{7}$ & orelha-de-elefante & Syngonium spp & Araceae & 3,35 \\
\hline $\mathbf{1 6}$ & azaléia & Rhododendron spp & Ericaceae & 2,87 \\
\hline $\mathbf{1 1}$ & jiboia & Scindapsus aureus Engl. & Araceae & 2,87 \\
\hline $\mathbf{4 0}$ & costela-de-Adão & Monstera deliciosa Liebm. & Araceae & 2,39 \\
\hline $\mathbf{7 5}$ & espada-de-são- jorge & Sansevieria trifasciata Hort ex Paine & Liliaceae & 2,39 \\
\hline $\mathbf{6 7}$ & mandioca-do-cerrado & Schefflera spp & Araliaceae & 2,39 \\
\hline $\mathbf{3 0}$ & vedélia & Sphagneticola trilobata (L.) Pruski & Asteraceae & 2,39 \\
\hline $\mathbf{2}$ & antúrio & Anthurium sp & Araceae & 1,91 \\
\hline $\mathbf{2 9}$ & azulzinha & Evolvulus sp & Convolvulaceae & 1,91 \\
\hline $\mathbf{7 8}$ & pingo-de-ouro & Duranta repens L. & Verbenaceae & 1,91 \\
\hline $\mathbf{3}$ & babosa-de-árvore & Philodendron martianum Engl. & Araceae & 1,44 \\
\hline $\mathbf{3 4}$ & bela-emília & Plumbago spp & Plumbaginaceae & 1,44 \\
\hline $\mathbf{6 8}$ & camarão-amarelo & Pachystachys lutea Nees & Acanthaceae & 1,44 \\
\hline $\mathbf{8 3}$ & ciclantus & Cyclanthus bipartitus Poit. ex A.Rich. & Cyclanthaceae & 1,44 \\
\hline $\mathbf{6 6}$ & dracena & Dracaena fragrans Ker Gawl. & Liliaceae & 1,44 \\
\hline $\mathbf{4 3}$ & gravatinha & Chlorophytum comosum Baker & Liliaceae & 1,44 \\
\hline $\mathbf{4 4}$ & lírio-do-amazonas & Eucharis grandiflora Planch. \& Linden & Amaryllidaceae & 1,44 \\
\hline $\mathbf{6}$ & palmeira-rápis & Rhapis excelsa Henry ex Rehder & Arecaceae & 1,44 \\
\hline $\mathbf{5}$ & planta-alumínio & Pilea cadierei Gagnep. \& Guillaumin & Urticaceae & 1,44 \\
\hline $\mathbf{1}$ & pleomele & Pleomele reflexa N.E.Br. & Liliaceae & 1,44 \\
\hline $\mathbf{2 4}$ & primavera & Bougainvillea glabra Choisy & Nyctaginaceae & 1,44 \\
\hline $\mathbf{3 1}$ & resedá & Lagerstroemia indica L. & Lythraceae & 1,44 \\
\hline $\mathbf{4}$ & zamiaculca & Zamioculcas amiifolia (Lodd.) Engl. & Araceae & 1,44 \\
\hline & & & & \\
\hline
\end{tabular}

Para as outras 14 espécies mais frequentes (30,59\% das populações nos canteiros), encontraram-se estudos científicos que indicam potencial de toxicidade, destacam-se espécies produtoras de cristais de oxalato de cálcio que, em contato com a mucosa digestiva podem provocar irritação com sensação de queimadura, salivação, edema dos lábios, língua e garganta, levando a obstrução das vias aéreas e até mesmo o óbito por insuficiência respiratória aguda. Distúrbios gastrointestinais, como cólicas, náusea e vômitos, também são frequentes. $E$ do contato do látex com a pele pode ocorrer dermatite de contato, especialmente na mucosa ocular, com irritação, edema, lacrimejamento, inflamação da córnea e conjuntiva (SIMÕES et al., 2001).

Segundo Simões e colaboradores (2001) as espécies da família Araceae possuem idioblastos com cristais de oxalato de cálcio no formato de agulhas (ráfides), que estão localizados em todas as partes da planta. 
Oito espécies tem potencial de toxicidade por apresentarem cristais de oxalato de cálcio: Spathiphyllum wallisii (lírio-da-paz), Syngonium spp (orelha-deelefante), Scindapsus aureus (jibóia), Monstera deliciosa (costela-de-adão), Schefflera spp (mandioca-do-cerrado), Anthurium sp (antúrio), Philodendron martianum (babosa-de-árvore) e Zamioculcas amiifolia (zamiaculca) (SIMÕES et al., 2001; e HARAGUCHI e CARVALHO, 2010); correspondendo a 19,14\% das populações que ocorrem nos canteiros.

Outra das espécies mais frequentes com potencial de toxicidade é a Rhododendron spp (azaléia - 2,87\% das populações vegetais) por apresentar diterpenos derivados do andromedano que possui ação cardiotóxica, que pode provocar salivação, vômitos, hipotensão, lacrimação, convulsões, bradicardia e coma (SIMÕES et al., 2001). Segundo Milewski e Khan (2006) pode também apresentar acetylandromedol e rhodotoxina, que em cães podem retardar a repolarização de fibras musculares ventriculares provocando bradicardia e taquicardia.

Recentemente foram isolados outros constituintes químicos presentes na azaléia como triterpenos e flavanona glicosilada, bem como dois ácidos rhodoaurichromanicos (A e B), combatentes do HIV, dihydroflavonol glicosilado, terpenoides diversos, iridoides glicosilados, entre outros (AHMAD et al., 2010).

Haraguchi e Carvalho (2010) verificaram que a Sansevieria trifasciata Hort ex Paine (espada-de-são-jorge - 2,39\% das populações vegetais), apresenta saponinas e compostos poli acetilênicos que, do contato com seu látex, podem irritar, provocar lesões ou processos alérgicos.

Duranta repens (pingo-de-ouro - 1,91\% das populações) apresenta esteroides nos frutos que da ingestão podem provocar febre, sono, dilatação da pupila, taquicardia, convulsões e gastroenterites (LOPES et al., 2009; SILVA, 2009).

Por sua vez, segundo Paulsen e colaboradores (2011), Pachystachys lutea (camarão-amarelo - 1,44\% das populações) pode provocar dermatite de contato especialmente da manipulação, tendo sido verificada esta toxicidade em jardineiros.

Sobre Bougainvillea glabra (primavera - 1,44\% das populações) Lopes e colaboradores (2009: 307) citando Bolognesi et al. (1997) afirmaram que: 
"A planta apresenta toxicidade para camundongos, devido à presença nas folhas de uma proteína inativadora de ribossomos do tipo I, a qual catalisa um dano irreversível ao rRNA, inibindo a síntese protéica. Esse dano corresponde à hidrólise de uma única ponte glicosídica em um resíduo de adenina altamente conservado. A dose letal desta proteína é superior a $32 \mathrm{mg} / \mathrm{kg} . "$

Matos e colaboradores (2011) afirmam que a literatura cita que a plumbagina, substância comum em Plumbago sp (bela-emilia - 1,44\% das populações), causa diarreia, náusea, vômito, febre, erupção na pele, danos no fígado, calafrios e anomalias sanguíneas.

Vale ressaltar a ocorrência de algumas espécies, dentre as 74 menos frequentes nos canteiros, que apresentam indicação de toxicidade nas obras científicas consultadas:

$\checkmark$ Codiaeum variegatum (cróton) possui nas sementes o alcalóide crotina, que com a ingestão de cinco sementes pode matar (SILVA, 2009);

$\checkmark$ Dieffenbachia picta (comigo-ninguém-pode) possui como princípio toxico oxalato de cálcio (HARAGUCHI e CARVALHO, 2010);

$\checkmark$ Buxus sempervirens (buxinho) apresenta óleo butiráceo volátil e alcaloides (buxina entre outros), se ingeridos causa distúrbios gastrointestinais, cólicas, náuseas vômitos e diarreia (BARG, 2004);

$\checkmark$ Euphorbia pulcherrima (bico-de-papagaio) possui ésteris diterpênicos, que não foram comprovados nesta espécie, porém o contato com o látex provoca reações semelhantes à Euphorbia millii (coroa-de-cristo) cuja ingestão pode provocar vômitos e diarreia; estudos recentes indicam a presença de mais princípios ativos, no seu látex, como pulcherol e um mono-N-acetil derivado do ácido alfagamadiaminobutírico, e nas folhas encontraram ácido cafeico, alfa-amirina, pseudotaraxasterol e beta-sinostrol; o látex é irritante para a pele e pode provocar inflamação ocular (OTAIZA et al., 2006);

$\checkmark$ Ficus spp (ficus) todas as partes da planta são toxicas apresenta glicosídeo cianogenético e seu látex são purgativos, levando a dermatites (SILVA, 2009);

$\checkmark$ Ficus pumila (unha-de-gato) contém no látex saponinas e compostos poliacetilênicos que irritam o local de contato provocando lesões e processos alérgicos (HARAGUCHI e CARVALHO, 2010);

$\checkmark$ Hemerocallis flava (lírio-comum) geralmente estas plantas são pertencentes a famílias Liliaceae e Amaryllidaceae que apresentam alcalóides amarilidáceos em 


\section{Periódica Eletrânica \\ Fórum Ambiental}

da Alta Paulista
Volume 11, Número 08, 2015

Saúde e Saneamento Ambiental

seus bulbos germinativos, que são cáusticos e quando ingeridos a degeneração hepática (HARAGUCHI e CARVALHO, 2010); estudos realizados em gatos com o lírio (Hemerocallis spp) indicam toxicidade principalmente por falha renal (anúria), desidratação, vômitos, diarreia e depressão; em alguns gatos ainda verificaram sinais no SNC como ataxia, desorientação e tremores; o tratamento indicado varia de uma hemodiálise até um transplante renal (MILEWSKI e KHAN, 2006);

$\checkmark$ Nerium oleander (espirradeira) utilizada como planta ornamental no Brasil possui todas as partes da planta tóxicas, especialmente folhas e sementes que possuem maior teor de ativos tóxicos, os glicosídeos cardioativos oleandrina e folinerina (OLIVEIRA e AKISUE, 2009); o mecanismo da oleandrina é inibir a bomba de sódio/potássio ATPase, evitando o transporte ativo de sódio, e permitindo a saída de potássio da célula (MILEWSKI e KHAN, 2006); os principais sintomas e mais graves são os transtornos neurológicos e cardíacos (semelhante aos digitálicos) levando ao coma e até a morte, porém outros sintomas iniciais podem ser esperados como diarreia, cólicas abdominais e náuseas (OLIVEIRA e AKISUE, 2009).

Todavia é importante ressaltar que, dentre as plantas mais frequentes nos canteiros do Campus Mooca da USJT, há espécies que apresentam indicação terapêutica:

$\checkmark$ Rhododendron spp (azaléia) apesar de apresentar constituintes tóxicos algumas espécies são utilizadas para tratamento de inflamação, dor, sintomas de resfriado, doenças de pele e distúrbios gastrointestinais (POPESCU e KOPP, 2013);

$\checkmark$ Sansevieria trifasciata (espada-de-são-jorge) na Malásia é muito utilizada para dor de ouvido, inchaço e febre, porém não há estudos que comprovem estas eficácias; Sunilson e colaboradores (2009) em um estudo, feito em ratos, com extratos da planta, mostrou que estes extratos apresentam analgesia leve e que o mecanismo de atividade possa ser de ação central;

$\checkmark$ Schefflera leucanta cuja infusão, segundo Potduang e colaboradores (2007), é utilizada para aliviar alergias, asma, tosse e infecções do trato respiratório e também a planta inteira é utilizada como analgésico e no processo de circulação sanguínea; já as folhas são utilizadas para cicatrização, tosse, bronquite dentre outros; estes autores verificaram que o principio ativo é broncodilatador e desta forma na Tailândia e China é utilizada como antiasmático; em alguns testes com Shefflera leucantha verificaram também agentes anti-cancêr, antioxidantes e atividade antimicrobiana; 
Sphagneticola trilobata (vedélia) é utilizada popularmente para dores lombares, dores musculares, reumatismo, ulcera, artrite entre outros; o ácido caurenóide e a luteína, presentes na planta, possuem atividade anticoncepcional; extratos contendo hexano e acetato de etila apresentaram atividade antimicrobiana tanto para grampositivos quanto gram-negativos (SILVA et al., 2012).

\section{CONCLUSÕES}

VI.

Verificou-se que nos 19 canteiros ornamentais presentes no Campus Mooca da USJT há 209 populações de vegetais, pertencentes à cerca de 100 espécies diferentes, e que mais da metade destas populações $(55,50 \%)$ correspondem a 26 espécies das quais 14 espécies (30,59\% das populações) apresentam potencial de toxicidade, especialmente se ingeridas diretamente ou se exsudatos que as constituem (como o látex, por exemplo) entrarem em contato com mucosas.

Considerando que o local de estudo apresenta enorme fluxo de pessoas entre funcionários, alunos e visitantes (em torno de 25.000 especialmente no período noturno) estes resultados podem contribuir para escolha de medidas de planejamento paisagístico que ofereçam maior segurança às pessoas.

\section{REFERÊNCIAS BIBLIOGRÁFICAS}

AHMAD, K.; REHMAN, S.; CHISTI, A. M.; SHAWL, A. S.; TANEJA, S. C.. Chemical constituents of Rhododendron lepidotum. Chemistry of Natural Compounds, v.46, n.2, 2010. Disponível em: http://link.springer.com/article/10.1007\%2Fs10600-010-9566-9\#page-1. Acesso em: 01 Abr. 2015.

BARCELLOS, D. C.. Plantas Tóxicas. Plantamed. sep, 2012. Disponível em: http://www.plantamed.com.br/DIV/Plantas toxicas.htm. Acesso em: 02 dez 2012.

BARG D. G. Plantas tóxicas. Instituto brasileiro de estudos homeopáticos. São Paulo, 2004. Disponível em: file://C:/Users/Jefferson/Downloads/Plantas toxicas\%20(1).pdf. Acesso em: 19 out. 2014.

BIONDI, D.; LEAL, L.; SCHAFFER, M.. Aspectos importantes das plantas ornamentais em escolas públicas estaduais da cidade de Curitiba, PR. Recife, PE. Revista Brasileira de Ciências Agrárias. v.3, n.3, p.267-275, jul-set, 2008. Disponível em: http://www.agraria.pro.br/sistema/index.php?journal =agraria\&page =article\&op=view\&path $\% 5 b \% 5 d=325 \&$ path $\% 5 b \% 5 d=185$. Acesso em: 02/12/2012.

FABIANO, P. O.; FRANCISCO O.. Plantas Toxicas: intoxicações causadas por espécies ornamentais devido a falta de informações, em Ourinhos -SP. In: VIII Congresso de Iniciação Cientifica das Faculdades Integradas de Ourinhos. Anais. Ourinhos: FIO/FEMM, 2009, 6p. Disponível em: http://fio.edu.br/cic/anais/2009 viii cic/Artigos/04/04.31.pdf. Acesso em: 13 dez 2012.

HARAGUCHI, L. M. M.; CARVALHO, O. B.. Plantas medicinais. São Paulo: Secretaria Municipal do verde e do meio ambiente. Divisão técnica escola municipal de jardinagem. 248p. 2010. 
LOPES, R. K.; RITTER, M. R. e RATES, S. M. K.. Revisão das atividades biológicas e toxicidade das plantas ornamentais mais utilizadas no Rio Grande do Sul, Brasil. R. bras. Bioci., Porto Alegre, v. 7, n. 3, p. 305-315, jul./set. 2009. Disponível em: http://www.ufrgs.br/seerbio/ojs/index.php/rbb/ article/viewFile/1056/878. Acesso em: 01 Abr. 2015.

LORENZI, H.; SOUZA, H. M.. Plantas ornamentais no Brasil: arbustivas herbáceas e trepadeiras. $2^{\circ}$ ed. Rev. e ampliada. Nova Odessa, SP: Instituto Plantarum, 1999. 1.088p.

SOUZA, V. C.; LORENZI, H.. Botânica Sistemática: guia ilustrado para identificação das famílias de fanerógamas nativas e exóticas no Brasil, baseado em APGIII. Nova Odessa, SP: Instituto Plantarum, 2008. 768p.

MATOS, F. J. A.; LORENZI H.; SANTOS, L. F. L.; MATOS, M. E. O.; SILVA, M. G. V.; SOUZA, M. P.. Plantas Tóxicas Estudo de Fitotoxicologia Química de Plantas Brasileiras. Nova Odessa/SP: Intituto Plantarum, 2011. 247p.

MILEWSKI, L. M.; KHAN, S. A.. An overviewof potentially life-threatening poisonous plants in dogs and cats. Journal of Veterinary Emergency and Critical Care. v.16, n.1, pp 25-33, 2006. Disponível em: DOI:10.1111/1.1476-4431.2005.00151.x. Acesso em: 01 Abr. 2015.

NUCCI, J. C.. Qualidade ambiental e adensamento urbano: um estudo de ecologia e planejamento da paisagem aplicado ao distrito de Santa Cecília (MSP) $2^{a}$ ed.. Curitiba: O Autor, 2008. 150 p.; il. Disponível em: http://www.labs.ufpr.br/site/arquivos/qldade_amb_aden_urbano.pdf. Acesso em: 20 Marc. 2015.

OLIVEIRA, F.; AKISUE, G.. Fundamentos de farmacobotânica e de morfologia vegetal. $3^{\circ}$ ed. São Paulo: Editora Atheneu, 2009.

ORTEGA, I. A. R.; MIGUEL, I; CARTILLONE M. R.; SILVA, M. J.. Companhia de desenvolvimento habitacional e urbano. 2008. Disponível em: http://www.cdhu.sp.gov.br/download/manuais-ecadernos/manual-de-paisagismo.pdf. Acesso em: 30/09/13.

OTAIZA, R. G.; ARZOLA, J. C.; ARREDONDO, M. C. R.. Estudio etnobotánico de espécies toxicas, ornamentales y medicinales de uso popula, presentes em El Jardín de Plantas Madicinales "Dr. Luis Ruiz Terán" de La Facultad de Farmacia y Bioanálisis de La Universidad de Los Andes. Boletín Antropológico, v.24, n.68, 463-481pp., septiembre-diciembre, 2006. Disponível em: http://www.redalyc.org/articulo.oa?id=71206805. Acesso em: 20 Marc. 2015.

POPESCU, R.; KOPP, B.. The genus Rhododendron: Anethnopharmacologicaland toxicologicalreview. Journal of Ethnopharmacology, n. 147, 42-62pp., 2013. Disponível em: http://dx.doi.org/10.1016/j.jep.2013.02.022. Acesso em: 11 abr 2015

PAULSEN, E., ANDERSEN, S. L. e ANDERSEN, K. E.. Occupational contact dermatitis from golden shrimp plant (Pachystachys lutea). Contact Dermatitis, n. 60, 293-294pp, 2011. Disponível em: doi:10.1111/j.1600-0536.2009.01531.x. Acesso em: 11 abr 2015.

POTDUANG, B.; CHONGSIRIROEG, C.; BENMART, Y.; GIWANON, R.; SUPATANAKUL, W.; TANPANICH, S.. Biological activities of Schefflera leucantha. Afr. J. Trad., CAM4, n.2: 157 - 164pp., 2007. Disponível em: http://www.ajol.info/index.php/ajtcam/article/view/31204/30538. Acesso em: 20 Marc. 2015.

SAlATINO A.. Nós e as Plantas: ontem e hoje. São Paulo, Revista Brasil. Bot. v.24, n. 4 (suplemento), p.483-490, dez. 2001.

SAMPAIO, L.. Historia de São $\quad$ Paulo. $2014 . \quad$ Disponível em:http://www.cidadedesaopaulo.com/sp/br/historia. Acesso em: 19/07/2014. 
SILVA L. C.. Plantas ornamentais tóxicas presentes no shopping Riverside Walk em Teresina - PI. Piracicaba - SP, REVSBAU, v.4, n.3, p.69-85, 2009. Disponível em: http://www.revsbau.esalq.usp.br/artigos_cientificos/artigo84.pdf Acesso em: 21 ago 2013.

SILVA, C. J.. BARBOSA, L. C. A.; DEMUNER, A. J.; MONTANARI, R. M.; FRANCINO, D.;MEIRA, R. M. S. A.; SOUZA, A. O.. Chemical composition and histochemistry of Sphagneticola trilobata essential oil. Brazilian Journal of Pharmacognosy, v. 22, n. 3, 482-489pp., May/Jun, 2012. Disponível em: http://www.scielo.br/pdf/rbfar/v22n3/aop00512.pdf. Acesso em: 20 Marc. 2015.

SILVA, I. G. R.; TAKEMURA O. S.. Aspectos de intoxicações por Dieffenbachia ssp (Comigoninguém-pode) - Araceae. Salvador, R. Ci. méd. biol., v. 5, n. 2, p. 151-159, mai./ago. 2006.

SIMÕES, C. M. O.; SCHENKEL, E. P.; GOSMANN G.; MELLO, J. C. P.; MENTZ L. A.; PETROVICK, P. R.. Farmacognosia: da planta ao medicamento. $3^{\circ} \mathrm{ed}$. Porto Alegre/Florianópolis: Ed. Universidade/UFRGS/Ed. Da UFSC, 2001.

SUNILSON A, J. J.; JAYARAJ, P.; VARATHARAJAN, R.; THOMAS, J.; JAMES, J.; MUTHAPPAN, M.. Analgesic and antipyretic effects of Sansevieria trifasciata Leaves. Afr. J. Trad., CAM6, n.4, 529533pp., 2009. Disponível em: http://www.ncbi.nlm.nih.gov/pmc/articles/PMC2816473/pdf/AJT06040529.pdf. Acesso em: 20 Marc. 2015. 